\title{
Patient-reported outcome measures in the recovery of adults hospitalised with community-acquired pneumonia: a systematic review
}

\author{
Harry J. Pick ${ }^{1}$, Charlotte E. Bolton ${ }^{2}$, Wei Shen Lim ${ }^{1,2}$ and Tricia M. McKeever ${ }^{3}$ \\ Affiliations: ${ }^{1}$ Dept of Respiratory Medicine, Nottingham University Hospital NHS Trust, Nottingham, UK. \\ ${ }^{2}$ NIHR Nottingham BRC Respiratory Theme, School of Medicine, University of Nottingham, Nottingham, UK. \\ ${ }^{3}$ Division of Epidemiology and Public Health, School of Medicine, University of Nottingham, Nottingham, UK.
}

Correspondence: H.J. Pick, Dept of Respiratory Medicine, Room B02, Clinical Sciences Building, Nottingham City Hospital Campus, Nottingham University Hospitals NHS Trust, Hucknall Road, Nottingham, NG5 1PB, UK. E-mail: harry.pick@nuh.nhs.uk

@ERSpublications

Patients report substantial morbidity in CAP recovery; the commonest symptom is fatigue (45-73\%), followed by cough (35-70\%) and dyspnoea (34-67\%). Methodological consistency is weak across studies. A core set of PROMs is suggested. http://ow.ly/d6N430nhdIq

Cite this article as: Pick HJ, Bolton CE, Lim WS, et al. Patient-reported outcome measures in the recovery of adults hospitalised with community-acquired pneumonia: a systematic review. Eur Respir J 2019; 53: 1802165 [https://doi.org/10.1183/13993003.02165-2018].

ABSTRACT Symptomatic and functional recovery are important patient-reported outcome measures (PROMs) in community-acquired pneumonia (CAP) that are increasingly used as trial end-points. This systematic review summarises the literature on PROMs in CAP.

Comprehensive searches in accordance with the PRISMA statement were conducted to March 2017. Eligible studies included adults discharged from hospital following confirmed CAP and reporting PROMs.

15 studies ( $n=5644$ patients) were included; most were of moderate quality. Studies used a wide range of PROMs and assessment tools. At 4-6 weeks post-discharge, the commonest symptom reported was fatigue (45.0-72.6\% of patients, three studies), followed by cough (35.3-69.7\%) and dyspnoea (34.2-67.1\%); corresponding values from studies restricted by age $<65$ years (two studies) were lower: fatigue $12.1-25.7 \%$, cough 19.9-31.9\% and dyspnoea 16.8-27.5\%. Functional impairment 4 weeks post-discharge was reported in $18-51 \%$ of patients (two studies), while median time to return to normal activities was between 15 and 28 days (three studies).

Substantial morbidity is reported by patients up to 6 weeks post-discharge. There is weak methodological consistency across existing studies. A core set of PROMs for use in future studies is suggested.

This article has supplementary material available from erj.ersjournals.com

This study is registered at PROSPERO with identifier number CRD42017059799.

Received: June 132018 | Accepted after revision: Dec 192018

Copyright OERS 2019 


\section{Introduction}

Community-acquired pneumonia (CAP) affects $\sim 1 \%$ of the UK adult population each year, accounting for over 100000 hospital admissions [1]. The average length of stay is 6 days and estimated direct UK healthcare costs are GBP441 million [2,3]. Most patients survive their inpatient admission and are discharged to recuperate $[4,5]$. In clinical practice, a patient is deemed to have recovered from CAP based on the clinical assessment of a physician, often in association with radiological improvement. In one study, $78 \%$ of patients were deemed clinically cured at 4 weeks following discharge from hospital [6], while corresponding rates of radiological resolution vary between $53 \%$ and $67 \%$ [6-8].

However, there is discordance between physician-rated clinical cure and radiographic resolution versus patient-reported symptoms and functional impairment [6]. Patient-reported outcome measures (PROMs) are increasingly recognised as providing a more robust indication of patient-experienced morbidity during recovery [9]. As a reflection of this, recent US Food and Drug Administration guidance on drug development in CAP recommends incorporation of PROMs as trial end-points [10]. The aim of this study was to systematically gather and summarise the available literature regarding PROMs following a hospital admission episode for CAP in order to inform future research in this area.

\section{Methods}

This systematic review was conducted and reported in accordance with the PRISMA statement [11]. The review protocol was prospectively registered in the PROSPERO database (identifier CRD42017059799).

\section{Search strategy}

Comprehensive searches of biomedical electronic databases were conducted: Cochrane Central Register of Controlled Trials (CENTRAL 2017, Issue 3), MEDLINE (1946 to March week 3, 2017), Embase (1980 to March 2017), CINAHL (1981 to March 2017), Allied and Complementary Medicine Database (AMED; 1985 to March 2017), and Web of Science (1985 to March 2017). The search strategy included subject headings and key words related to CAP, symptom recovery, functional activity, healthcare utilisation and treatment outcome. The searches were not subject to any language restrictions. Details of the search strategy for each database are given in the supplementary material. An additional grey literature search was conducted using Google Scholar to identify unpublished data of potential significance to the review. Reference lists of included studies were reviewed to identify potentially relevant articles. The full texts of identified articles were reviewed and assessed against eligibility criteria. Conference proceedings from 2014 to 2017 for the American Thoracic Society Conference, British Thoracic Society Winter Meeting and European Respiratory Society Congress were reviewed to identify potentially eligible articles.

\section{Study selection}

Randomised controlled trials (RCTs), quasi-experimental and nonrandomised studies in adults attending secondary care facilities with a presenting diagnosis of CAP and assessing at least one of the predefined patient-reported markers of recovery were included. A diagnosis of CAP was defined as: symptoms and signs consistent with an acute lower respiratory tract infection associated with radiological confirmation of pneumonia on chest radiography. Attendance at a secondary care facility was defined as any patient receiving their initial review and all or part of their treatment for CAP at an acute hospital facility, including emergency department visits, with or without subsequent admission.

Two study authors (H.J.P and T.M.M.) conducted a sequential review of identified studies independently, proceeding from title to abstract to full texts, with exclusions at each stage if studies did not fulfil eligibility criteria. Disagreement was resolved by discussion and consensus, involving a third review author (W.S.L.) as necessary.

\section{Outcome measures}

Primary outcomes of interest measured within 6 weeks following hospital discharge were: 1) patient-reported symptoms, 2) return to work and/or usual activities of daily living (ADLs), and 3) healthcare utilisation. Secondary outcomes of interest measured within 6 weeks following discharge were: 1) proportion with physician-determined clinical cure in studies reporting symptomatic and functional recovery, and 2) change in quality of life scores. (The time-point of 6 weeks after discharge was chosen for outcomes assessment because we anticipated most studies would report outcomes at that time-point and it coincides with the time-point at which other outcomes are commonly measured in studies of CAP.)

\section{Data extraction}

All data were independently extracted by two authors (H.J.P. and T.M.M.) using a pre-designed form that was initially piloted on five studies; any disparities were resolved by discussion and consensus, including a third reviewer (W.S.L.) as required. Where data were represented graphically within studies, Digitizelt 
version 2.3.3 (www.digitizeit.de) was used to derive estimated values. Information on study population, outcome and study design were collected.

Assessment of risk of bias

A specific quality assessment tool was created combining relevant aspects from the Newcastle-Ottawa scale and the Downs and Black Quality Assessment Tool $[12,13]$. Each study was assessed for details pertaining to study period and location, participant selection, and outcome measure, with a final total score out of 6 points (with 0 representing low quality and 6 representing the highest possible quality rating.)

\section{Data synthesis}

Extracted results were reviewed to assess if adequate similarity existed with respect to outcomes to conduct a random effects meta-analysis using Stata version 15 (StataCorp, College Station, TX, USA). The $I^{2}$ statistic was used to assist with assessment of heterogeneity between studies.

\section{Results}

The search strategy identified 3958 articles, of which 15 articles fulfilled the eligibility criteria and were included in the systematic review (see study flow diagram in figure 1) [6, 14-27].

\section{Characteristics of included studies}

The characteristics of the 15 included studies are summarised in table 1. Briefly, eligible studies included two RCTs, eight prospective cohort studies, one retrospective cohort study and four studies with cohort

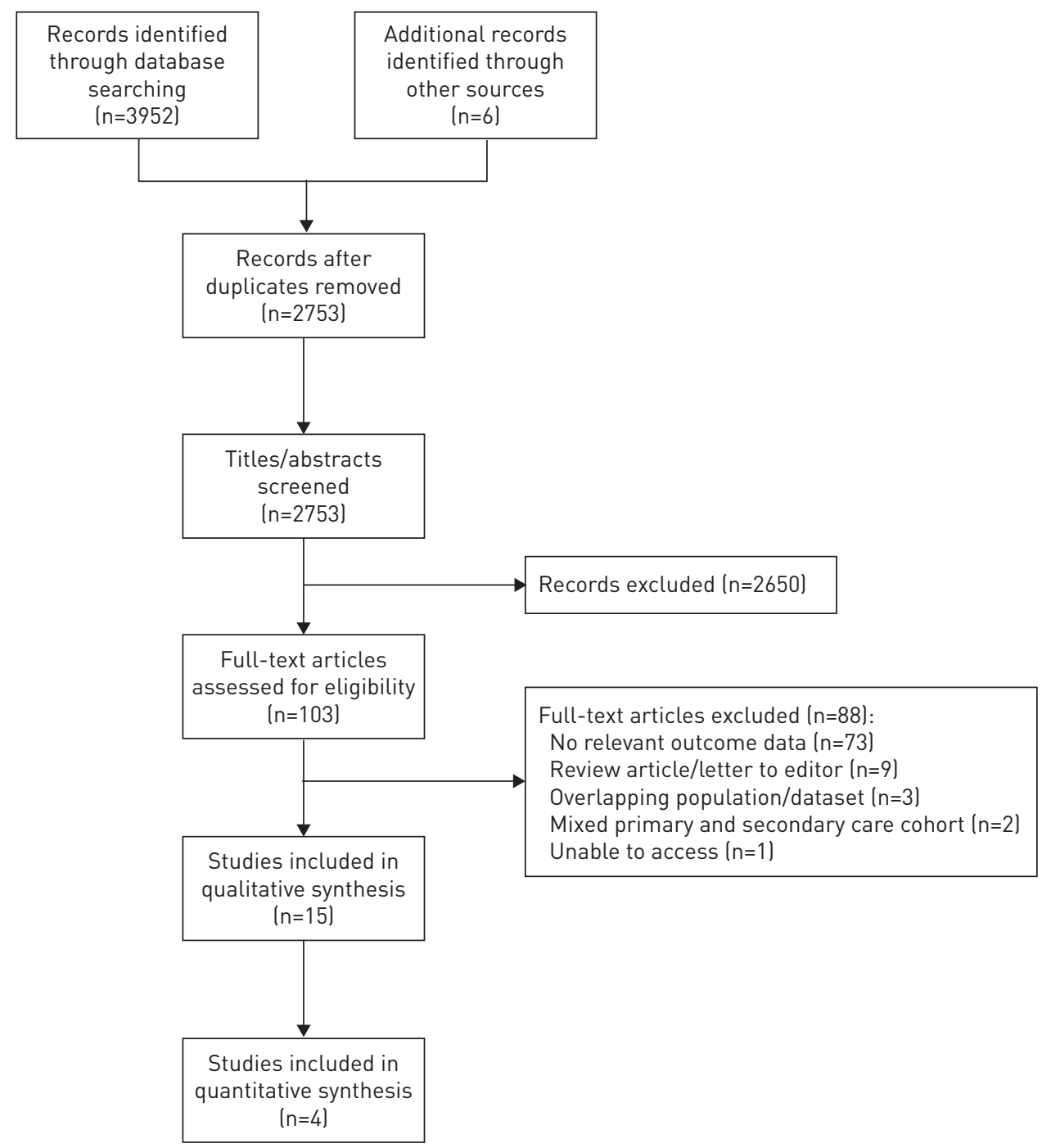

FIGURE 1 Study flow diagram. 
TABLE 1 Summary of included studies

First author [ref.] Country Study period Study typ

Study population

Outcome

Studies with participants selected for low/moderate severity CAP or age $<65$ years ( $n=6$ studies, $n=1217$ individual participants)

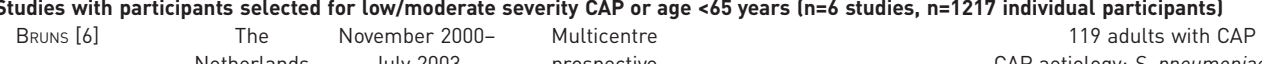

$\begin{array}{ccc}\text { CARRATALA [16] Spain } \quad \begin{array}{c}\text { October 2000- } \\ \text { October 2002 }\end{array} & \begin{array}{c}\text { Cohort from } \\ \text { multicentre RCT }\end{array}\end{array}$

\begin{tabular}{|c|c|c|}
\hline Daniel [17] & UK & $\begin{array}{l}\text { February 2015- } \\
\text { April } 2016\end{array}$ \\
\hline
\end{tabular}

EL Moussaoul [18] The November 2000- Cohort from Netherlands July 2003 multicentre RCT

LABERERE [20] USA/ January 2001- Cohort from

Canada December 2001 multicentre RCT

Selection criteria: age: unselected (median (IQR) age 66 (48-77) years); severity: low severity, PS classes I-III (PSI I: 16.0\%; PSI II: 41.0\%; PSI III: 43.0\%); comorbid disease: excluded if pulmonary alcoholism and evidence of end-organ damage, social problems incompatible with recruitment, illicit drug use 30 days; immune status: immunocompetent only lexcluded if HIV. immunosuppression!

MetLaY [22] USA April 1996- $\quad$ Single-centre

prospective

126 adults with CAP (male 54.8\%)

CAP aetiology: S. pneumoniae 14.0\%, L. pneumophila 4.4\%, H. influenzae 2.6\%, "atypical" $2.6 \%$ Selection criteria: age: unselected (mean \pm so age: outpatient group $67.5 \pm 11.8$ years; inpatient

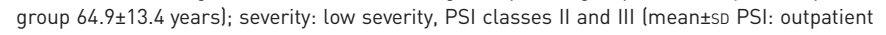

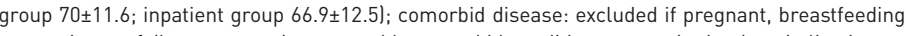
respiratory failure, concomitant unstable comorbid conditions necessitating hospitalisation,

complicated pleural effusion, lung abscess, shock, metastatic infection, severe social problems, splenectomy transplant); other: excluded if quinolone allergy or quinolone therapy in preceding 3 month

108 adults with CAP (male 48.2\%)

Selection criteria: age: 18-65 years only (median (IQR) age 50 (38.3-57.8) years); severity: unselected (CURB-65 0-1: 88.9\%; CURB-65 2: 9.3\%; CURB-65 ₹3: 1.8\%); comorbid disease: unselected; immune status: unselected

\section{1 adults with CAP (male 58.8\%)}

Selection criteria: age: unselected (median (IQR) age 65 (48-72) years); severity: low severity, PS $<110$ (mean \pm SD PSI 71 \pm 23 ); comorbid disease: excluded if pregnant, severe underlying disease, preceding antibiotic treatment for $>24 \mathrm{~h}$ prior to admission, concurrent comorbid disease likely interfere status: unselected; other excluded if amoxicillin allergy

\section{9 adults with CAP (male 44\%)}

Selection criteria: age: 18-64 years; severity: low severity, PSI classes I-III (mean PSI 55.2); comorbid disease: excluded if pregnancy, severe neuromuscular disease; immune status: immunocompetent only (excluded if chronic immunosuppression, HIV); other: nursing home residence, psychological or social problems compromising follow-up

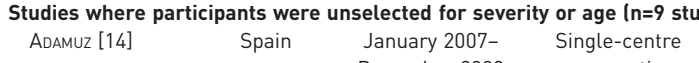 December 2009}

prospective

828 adults with CAP (male 65.8\%)

CAP aetiology: S. pneumoniae $44.1 \%$, L. pneumophila $3.0 \%$, H. influenzae $4.4 \%$, anaerobes $5.6 \%$ Selection criteria: age: unselected (median (IQR) age 71 (55-79) years); severity: unselected (PSI $>90$ in $62.2 \%$ ); comorbid disease: unselected; immune status: immunocompetent only (excluded HIV , splenectomy, immunosuppressive therapy, corticosteroid therapy equivalent to prednisolone $>20 \mathrm{mg}$ daily, neutropenia, Ig deficiency, solid organ transplantl) other: excluded if inpatient death

207 adults with CAP (male 59.9\%)

AP aetiology: S. pneumoniae $24.6 \%$, H. influenzae $5.4 \%$, influenzae A $3.4 \%$ Selection criteria: age: unselected (21-49 years: $20.2 \%$; $50-69$ years: $30.5 \%$; $>70$ years: $49.3 \%$ ); severity: unselected (CURB-65 0-1: 49.3\%; CURB-65 2: 34.3\%; CURB-65 ₹3: 16.4\%); comorbid
Normalisation of CAP score at day 10: $32.0 \%$; physician-rated clinical cure at day $28: 88.9 \%$

SF-36 score: "At 30-day follow-up SF-36 scores remained abnormal, but had returned towards baseline for Spanish population"

Proportion of patients reporting prevalence of symptoms and functional impairment at 28 days following discharge: cough $31.9 \%$ dyspnoea $27.5 \%$, chest pain $16.5 \%$, sputum $11.0 \%$, fatigue $12.1 \%$, $\geqslant 1$ symptom $58.2 \%$, not returned to ADLs $51.1 \%$, not returned to work 34.3\%; reconsultation within 28 days: ED attendance $12.0 \%$, primary care $59.2 \%$

Median (IQR) CAP scores: day 3, 56 (38-69); day 7, 60 (37-75); day 10,65 (37-86); day 14, 77 (61-88); day 28, 75 (58-93)

Median (IQR) time for return to ADLs: workers 22 (11-29) days, Median (IQR) (ime for return to ADLs: workers $22(11-29)$ days, 29) days

Proportion of patients reporting prevalence of symptoms at 28 days following discharge: cough $19.9 \%$, dyspnoea $16.8 \%$ fatigue $25.7 \%$,

$$
\text { fever } 3.5 \%, \geqslant 1 \text { symptom } 35.0 \%
$$

Healthcare utilisation at 30 days: ED 21.4\%, primary care $18.0 \%$; symptoms reported in participants reconsulting within 30 days: primary care (n=149): respiratory symptoms $75.2 \%$, general symptoms $228 \%$. ED (n=177): worsening pneumonia symptoms $47.5 \%$

Healthcare utilisation at 30 days: primary care $20.3 \%$, ED $18.4 \%$; median (IQR) time off work: intervention group 30 (15-66.5) days, usual care group 26 (12.5-37) days 
TABLE 1 Continued resident or long-term care facility, language barrier

$\begin{array}{cccc}\text { FinE [19] } & \begin{array}{c}\text { USA/ } \\ \text { Canada }\end{array} & \begin{array}{c}\text { October 1991- } \\ \text { March 1994 }\end{array} & \begin{array}{c}\text { Multicentre } \\ \text { prospective } \\ \text { cohort }\end{array} \\ \text { MARRIE [21] } \quad \text { Canada } & \begin{array}{c}\text { January 1998- } \\ \text { July 1998 }\end{array} & \begin{array}{c}\text { Multicentre } \\ \text { prospective } \\ \text { cohort }\end{array} \\ \text { NicKLER [23] Switzerland } & \begin{array}{c}\text { October 2006- } \\ \text { March 2008 }\end{array} & \begin{array}{c}\text { Cohort from } \\ \text { multicentre RCT }\end{array}\end{array}$

1343 adults with CAP (male $52.4 \%$ )

CAP aetiology: S. pneumoniae 9.1\%, H. influenzae 4.8\%, "atypical" 2.1\%, Enterobacter sp. $2.8 \%$, P. aeruginosa $0.9 \%$

Selection criteria: age >65 years 58.7\%; severity: unselected (PSI I: 13.8\%; PSI II: 17.4\%; PSI III: 18.9\%; PSI IV: $33.2 \%$; PSI V: $16.7 \%)$; comorbid disease: unselected; immune status: excluded if HIV

535 adults with CAP (male 52.3\%) CAP aetiology: not reported

Selection criteria: age: unselected (mean \pm SD age $61.6 \pm 19.1$ years); severity: unselected (mean \pm S PSI 76.2 32.8 ; ; comorbid disease: unselected; immune status: unselected

\section{3 adults with CAP (male $58.8 \%$} CAP aetiology: not reported

Selection criteria: age: unselected (median (IQR) age 72 (52-82) years); severity: unselected (PS I: 10.0\%; PSI II: 19.7\%); comorbid disease: excluded if terminal illness; immune status:

immunocompetent only (excluded if long-term immunosuppression); other: excluded if languag impairment precluding written consent, i.v. drug abuse

Sharma [24] USA $\begin{array}{cc}\text { March 1995- } \\ \text { March 1998 }\end{array} \quad \begin{gathered}\begin{array}{c}\text { Single-centre } \\ \text { prospective }\end{array} \\ \text { cons }\end{gathered}$

\section{9 adults with CAP Imale $35 \%$} CAP aetiology: not reported

Selection criteria: age: unselected (meantsD age $79.9 \pm 6.1$ years); severity: unselected (mean \pm 50 PSI 106.2 +23.91 : comorbid disease: excluded if terminal illness; immune status: unselected; other: excluded if coma, intensive care admission, unable to participate in interview

\section{2 adults with CAP (male $62.8 \%$}

Selection criteria: age: unselected Imean士sD age $62.2 \pm 6.07$ and $64.7 \pm 18.7$ years in the control and intervention groups, respectively); severity: unselected (mean \pm sD PSI 83.7 \pm 33.7 and $81.8 \pm 33.8$, in the control and intervention groups, respectively); comorbid disease: unselected; immune status: immunocompetent only lexcluded if HIV, immunosuppression for solid organ transplantation, splenectomy, receiving $\geqslant 10 \mathrm{mg} \cdot$ day $^{-1}$ prednisone equivalent for $>30$ days, taking other immunosuppressive agents, neutropenia); other: excluded if care home resident, antibiotics

within preceding 30 days, intercostal chest drain, extrapulmonary manifestations, death or ICU

\section{9 adults with CAP (male 52\%)} CAP aetiology: not reported

WootTon [26] UK February 2011- $\quad \begin{aligned} & \text { Multicentre } \\ & \text { Mrospective }\end{aligned}$

Selection criteria: age: unselected (mean \pm SD age 68 (range 16-98) years); severity: unselected (CURB-65 0-1: 46.7\%; CURB-65 2: 29.6\%; CURB-65 $\geqslant 3: 23.7 \%$ ); comorbid disease: excluded if bronchiectasis, cystic fibrosis, advanced malignancy; immune status: unselected; other: excluded if palliative treatment, invasive ventilation

WYRWIC [27] USA Unknown $\begin{gathered}\text { Multicentre } \\ \text { retrospective }\end{gathered}$

\section{1 adults with CAP (male 45\%)}

CAP aetiology: not reported CAP aetiology: not reported
Selection criteria: age: $\geqslant 50$ years; severity: unselected; comorbid disease: unselected; immune
status: unselected; other: respondent to e-mail survey, excluded if nursing home resident
Proportion of patients reporting prevalence of symptoms and functional impairment at 30 days following discharge: cough $47.1 \%$ dyspnoea $46.5 \%$, sputum $42.3 \%$, fatigue $72.6 \%$, $\geqslant 1$ symptom $68.5 \%$; not returned to ADLs: workers $18 \%$ Imedian 15 daysl, nonworkers $42.8 \%$ (median 24 days); not returned to work $31.9 \%$

Proportion of patients reporting prevalence of symptoms/functional impairment at $14 / 42$ days following discharge: cough $55.8 \% / 35.3 \%$, dyspnoea $48.6 \% / 34.2 \%$, chest pain $17.3 \% / 11.6 \%$, sputum $35.9 \%$ / $26.4 \%$, fatigue $66.7 \% / 45.0 \%$, fever $8.4 \% / 4.7 \%$, $\geqslant 1$ symptom $85.6 \% /$ $64.3 \%$

EQ-5D index: decline in ADLs in $29 \%$ at day 30

Proportion with decline in ADLs score of $>1$ point $28 \%$ at day 30

Mean \pm SD CAP-symptom score at 5 days $24.7 \pm 11.4$ to $27.2 \pm 12.5$; mean \pm so CAP-symptom score at 10 days $18.6 \pm 9.0$ to $17.9 \pm 7.6$; median (IOR) time for return to normal activity $15(10-21)$ to

$$
18 \text { (9-25) days }
$$

Average CAP-symptom score 23.8 at day 2; average CAP-symptom score 13.6 at day 28; proportion of patients reporting prevalence of symptoms at 28 days following discharge: cough $13.6 \%$, dyspnoea $67.1 \%$, chest pain $31.2 \%$, fatigue $57.0 \%$, $\geqslant 1$ symptom $96.4 \%$

Mean (median (IQR)) time to symptom resolution: cough 13.6 (7 (2-14)) days, dyspnoea 25.1 (14 (7-43)) days, chest pain 12.9 fever 2.7 (2) sutum $14.8(7(7-14))$ days, fatigue $25(20(7-24))$ days. fever 2.7 (2 (1-2)) days; median time for return to work 14 days

CAP: community-acquired pneumonia; RCT: randomised controlled trial; S. pneumoniae: Streptococcus pneumoniae; PSI: Pneumonia Severity Index; L. pneumophila: Legionella pneumophila; H. influenzae: Haemophilus influenzae; SF-36: Short Form-36 health survey; IQR: interquartile range; CURB-65: confusion, urea $>7$ mmol.L-1, respiratory rate $\geqslant 30$ breaths $\mathrm{min}^{-1}$, blood pressure $<90 \mathrm{mmHg}$ (systolic) or $\leqslant 60 \mathrm{mmHg}$ (diastolic), age $\geqslant 65$ years; $P$. aeruginosa: Pseudomonas aeruginosa; ICU: intensive care unit; ADLs: activities of daily living; ED: emergency department; EQ-5D: EuroQoL health-related quality of life questionnaire. 
data for participants originally enrolled in RCTs. Six studies were restricted to patients with low/moderate severity CAP (Pneumonia Severity Index (PSI) classes I-III, PSI score $<110$ or CURB-65 (confusion, urea $>7 \mathrm{mmol} \cdot \mathrm{L}^{-1}$, respiratory rate $\geqslant 30$ breaths $\cdot \mathrm{min}^{-1}$, blood pressure $<90 \mathrm{mmHg}$ (systolic) or $\leqslant 60 \mathrm{mmHg}$ (diastolic), age $\geqslant 65$ years) score $0-2)[6,16-18,20,22]$ or age $<65$ years $[17,22]$. One study did not assess disease severity [27] and the remaining studies included patients of all disease severities, with mean PSI across studies ranging from $76.2 \pm 32.8$ to $106.2 \pm 23.9[14,15,19,21,23-26]$. Outcome measures of interest varied substantially between the included studies, with variable choice of outcome, tool for assessment and timing of measurement. Individual study methodology is summarised in table 2.

\section{Risk of bias in included studies}

The median (interquartile range (IQR)) quality score across all included studies was 4.5 (4-5). Three studies scored full marks at quality assessment (supplementary table S1) [15, 16, 25]. All included studies defined their follow-up period, with all but a single study adequately defining study population and study period. The study populations were often restricted by selection criteria, such as age, severity of disease, level of dependency or residential status (table 1), but these selection criteria were deemed appropriate when study design and outcomes of interest were considered. Included studies rarely reported loss to follow-up.

\section{Patient-reported symptoms}

Six studies included data for patient-reported prevalence of symptoms within 6 weeks of discharge [14, 17, $19,21,22,26]$.

Four studies reported prevalence of symptoms for participants unselected for age or disease severity $[14,19,21,26]$. At $28-42$ days post-discharge, the commonest symptom reported was fatigue (range 45.0$72.6 \%$ of patients), followed by cough (range 35.3-69.7\%) and dyspnoea (range 34.2-67.1\%) (figure 2) $[19,21,26]$. One study of patients attending for healthcare review within 30 days of discharge observed that respiratory symptoms were reported in $75.2 \%$ of those attending primary care and $47.5 \%$ of those attending emergency departments [14]. Restricting the population to working adults, two studies reported symptom prevalence at 4 weeks following discharge and found one or more symptom was reported by $35.0 \%$ and $58.2 \%$ of participants, cough by $19.9 \%$ and $31.9 \%$, dyspnoea by $16.8 \%$ and $27.5 \%$, and fatigue by $12.1 \%$ and $25.7 \%[17,22]$. Chest pain $(16.5 \%)$ and sputum production $(11 \%)$ were reported by participants in one of these studies [17].

Finally, in an e-mail survey investigating average time for resolution of symptoms following CAP in adults aged $\geqslant 50$ years $(n=500)$, median (IQR) time for resolution of cough was $7(2-14)$ days, dyspnoea 14 (7-43) days, chest pain 7 (2-14) days, sputum production 7 (7-14) days, fever 2 (1-2) days and fatigue 20 (7-24) days [27].

\section{Quantitative synthesis}

Random effects meta-analysis was performed on amenable data from four studies ( $\mathrm{n}=1715)$ reporting outcomes at 4 weeks $[17,19,22,26]$. High levels of heterogeneity were evident. The summary estimate of the proportion of patients reporting one or more symptoms was $70 \%$ (95\% CI $53-86 \% ; I^{2}=98.6 \%$ ), cough $42 \%$ (95\% CI $24-60 \% ; I^{2}=97.1 \%$ ), dyspnoea 39\% (95\% CI $\left.21-58 \% ; I^{2}=97.5 \%\right)$ and fatigue $42 \%$ (95\% CI $10-74 \% ; I^{2}=99.2 \%$ ) (figure $3 \mathrm{a}-\mathrm{d}$ ).

\section{CAP-specific symptom scores}

Four studies included data derived from pneumonia symptom scores $[6,18,25,26]$.

Two studies ( $\mathrm{n}=91$ and $\mathrm{n}=95$ ) used the CAP score (higher score signifying fewer symptoms). The median (IQR) CAP scores reported by EL Moussaour et al. [18] at days 3, 7, 10, 14 and 28 following admission were 56 (38-69), 60 (37-75), 65 (37-86), 77 (61-88) and 75 (58-93), respectively. BRuNs et al. [6] reported that normalisation of CAP score, relative to retrospectively assessed baseline scores 6 weeks prior to admission, occurred in $32 \%$ of patients at 10 days following hospital admission and $41.8 \%$ of patients at 28 days.

Two studies ( $\mathrm{n}=169$ and $\mathrm{n}=312$ ) used the CAP-symptom score (lower score signifying fewer symptoms). WootTon et al. [26] observed the average CAP-symptom score at day 2 from admission was 23.8 and at day 28 was 13.6. URANGA et al. [25], in an interventional trial of short-course antibiotics, reported that the average CAP-symptom score in the control group at day 5 from admission was $24.7 \pm 11.4$ and at day 10 was 18.6 \pm 9.0 , with corresponding values in the intervention group of $27.2 \pm 12.5$ and 17.9 \pm 7.6 .

\section{Return to functional activity}

Five studies included data for time to return to normal activities [15, 17, 19, 20, 25]. 


\begin{tabular}{|c|c|c|c|c|c|c|c|c|c|c|c|c|c|c|c|c|c|c|c|}
\hline \multirow[t]{3}{*}{ First author [ref.] } & \multirow{2}{*}{\multicolumn{2}{|c|}{$\begin{array}{l}\text { Destination } \\
\text { after initial } \\
\text { review }\end{array}$}} & \multirow{2}{*}{\multicolumn{2}{|c|}{ Severity }} & \multicolumn{10}{|c|}{ Outcome measure } & \multirow{2}{*}{\multicolumn{5}{|c|}{$\begin{array}{c}\text { Timing of } \\
\text { assessment of } \\
\text { outcome measure }\end{array}$}} \\
\hline & & & & & \multicolumn{3}{|c|}{$\begin{array}{l}\text { Assessment of } \\
\text { symptoms }\end{array}$} & \multicolumn{2}{|c|}{ Health status } & \multicolumn{2}{|c|}{$\begin{array}{l}\text { Assessment of } \\
\text { function } \\
\text { (return to) }\end{array}$} & \multirow[t]{2}{*}{$\begin{array}{l}\text { Clinical } \\
\text { cure }\end{array}$} & \multicolumn{2}{|c|}{$\begin{array}{l}\text { Healthcare } \\
\text { utilisation }\end{array}$} & & & & & \\
\hline & IP & $\mathrm{OP}$ & CURB-65 & $\overline{\text { PSI }}$ & Prevalence & $\begin{array}{l}\text { CAP } \\
\text { score }\end{array}$ & $\begin{array}{l}\text { CAP- } \\
\text { symptom } \\
\text { score }\end{array}$ & SF-36 & $E Q-5 D$ & ADLs & Occupation & & $\begin{array}{l}\text { Primary } \\
\text { care }\end{array}$ & ED & $\begin{array}{l}\text { Average } \\
\text { time to ... }\end{array}$ & $\begin{array}{l}\leqslant 14 \\
\text { days }\end{array}$ & $\begin{array}{c}28 \\
\text { days }\end{array}$ & $\begin{array}{c}30 \\
\text { days }\end{array}$ & $\begin{array}{c}42 \\
\text { days }\end{array}$ \\
\hline \multicolumn{20}{|c|}{ Studies with participants selected for low/moderate severity CAP or age $<65$ years ( $n=6$ studies, $n=1217$ individual participants) } \\
\hline BRUNS [6] & $\checkmark$ & & & $\checkmark$ & & 5 & & & & & & $\checkmark$ & & & & $\checkmark$ & $\checkmark$ & & \\
\hline Carratala [16] & $\checkmark$ & $\checkmark$ & & $\checkmark$ & & & & $\checkmark$ & & & & & & & & & & $\checkmark$ & \\
\hline Daniel [17] & $\checkmark$ & & 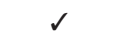 & & $\checkmark$ & & & & & $\checkmark$ & $\checkmark$ & & $\checkmark$ & $\checkmark$ & & & 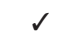 & & \\
\hline El Moussaoul [18] & d & & & d & & 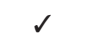 & & & & & & & & & & 2 & 1 & & \\
\hline LABERERE [20] & $\checkmark$ & $\checkmark$ & & $\checkmark$ & & & & & & $\checkmark$ & $\checkmark$ & & & & $\checkmark$ & & & & \\
\hline MetLay [22] & & $\checkmark$ & & $\checkmark$ & 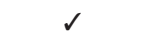 & & & & & & & & & & & & $\checkmark$ & & \\
\hline \multicolumn{20}{|c|}{ Studies where participants were unselected for severity or age ( $n=9$ studies, $n=4427$ individual participants) } \\
\hline Adamuz [14] & $\checkmark$ & & & $\checkmark$ & $\checkmark$ & & & & & & & & $\checkmark$ & $\checkmark$ & & & & $\checkmark$ & \\
\hline Adamuz [15] & 2 & & 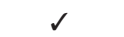 & & & & & & & & $\checkmark$ & & 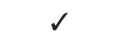 & $\checkmark$ & 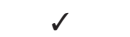 & & & 2 & \\
\hline FINE [19] & $\checkmark$ & $\checkmark$ & & $\checkmark$ & $\checkmark$ & & & & & $\checkmark$ & $\checkmark$ & & & & & & & $\checkmark$ & \\
\hline MARRIE [21] & $\checkmark$ & $\checkmark$ & & $\checkmark$ & $\checkmark$ & & & & & & & & & & & $\checkmark$ & & & $\checkmark$ \\
\hline NICKLER [23] & $d$ & & & $d$ & & & & & $d$ & & & & & & & & & $d$ & \\
\hline SHARMA [24] & $\checkmark$ & & & $\checkmark$ & & & & & & $\checkmark$ & & & & & & & & $\checkmark$ & \\
\hline URANGA [25] & $\checkmark$ & & & $\checkmark$ & & & $\checkmark$ & & & $\checkmark$ & & & & & $\checkmark$ & $\checkmark$ & & & \\
\hline Wootton [26] & $\checkmark$ & & $s$ & & $\checkmark$ & & 2 & & & & & & & & 2 & & $s$ & & \\
\hline WYRWICH [27] & $\checkmark$ & $\checkmark$ & & & $\checkmark$ & & & & & & $\checkmark$ & & & & $\checkmark$ & & & & \\
\hline
\end{tabular}



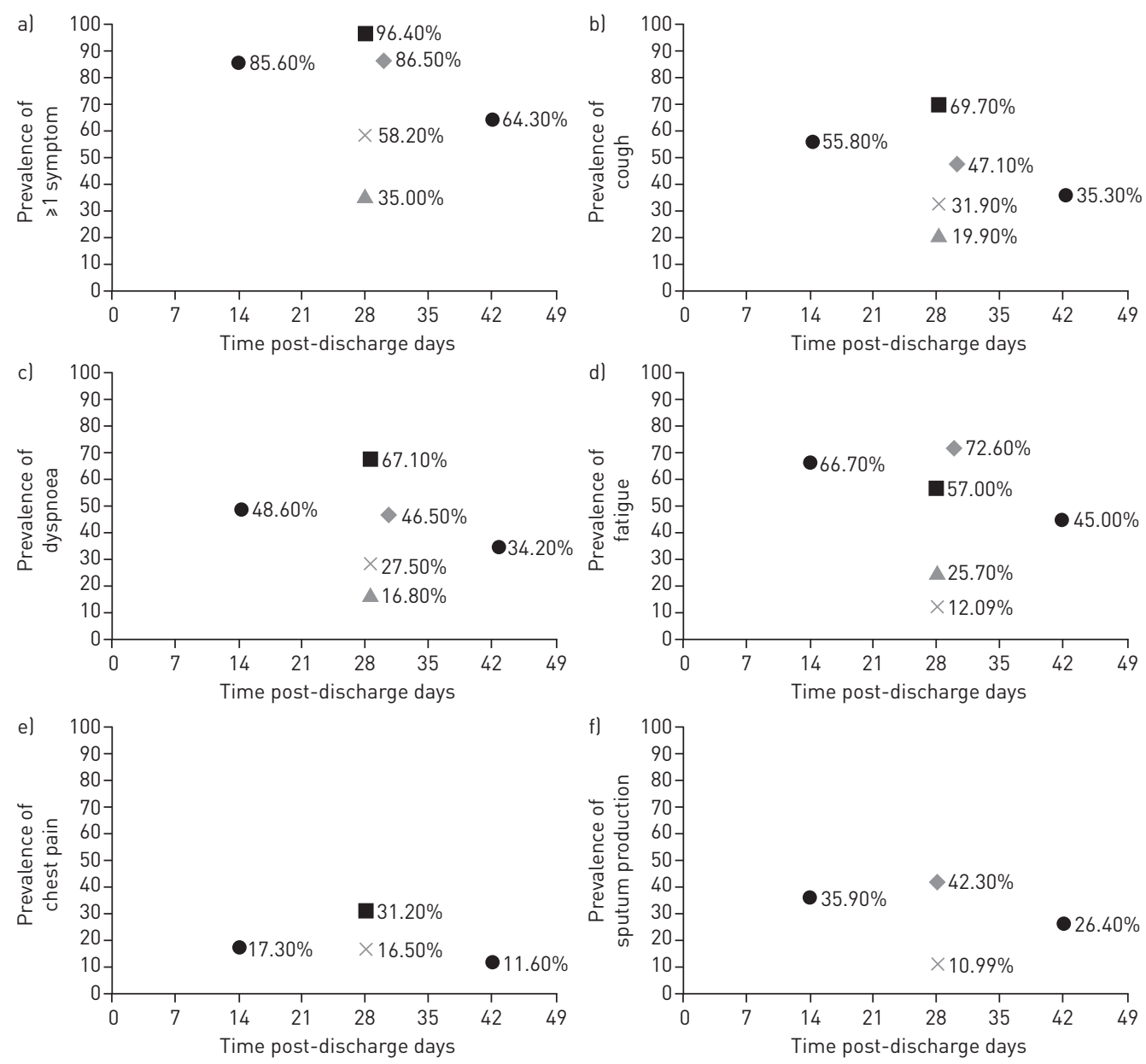

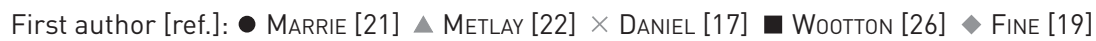

FIGURE 2 Prevalence of symptoms in recovery following community-acquired pneumonia (CAP): a) one or more symptom, b) cough, c) dyspnoea, d) fatigue, e) chest pain and f) sputum production. MEtLAY et al. [22] and DANIEL et al. [17] are studies of patient cohorts restricted to either low/moderate severity CAP (Pneumonia Severity Index classes I-III or CURB-65 (confusion, urea $>7 \mathrm{mmol} \cdot \mathrm{L}^{-1}$, respiratory rate $\geqslant 30$ breaths $\cdot \mathrm{min}^{-1}$, blood pressure $<90 \mathrm{mmHg}$ (systolic) or $\leqslant 60 \mathrm{mmHg}$ (diastolic), age $\geqslant 65$ years) score 0-2) or age <65 years, by design. In the study by MARRIE et al. [21], symptom prevalence was assessed twice during the follow-up period (at 14 and 42 days); the other studies reported symptom prevalence once in the defined follow-up period.

DANIEL et al. [17] found that $51.1 \%$ of adults aged $<65$ years had not resumed baseline ADLs at 4 weeks. In contrast, FINE et al. [19] found that $18.0 \%$ of workers had not resumed baseline ADLs at 4 weeks compared with $42.8 \%$ of nonworkers.

The median (IQR) time for return to normal activity reported by URANGA et al. [25] was 18 (9-25) days in the control group and 15 (10-21) days in the intervention group. Fine et al. [19] found the median time for return to ADLs in workers was 15 days and in nonworkers was 24 days, whereas LABARERE et al. [20] found little difference in the median (IQR) time for return to ADLs between workers (22 (11-29) days) and nonworkers (20 (9-29) days).

Of two studies with relevant data, 34.3\% [17] and 31.9\% [19] of participants had not returned to work at 4 weeks. Median time for return to work following discharge was 14 days [20, 27]. ADAmUz et al. [15] reported that the median (IQR) time taken off work by participants in the control group was 26 (12.5-37) days compared with 30 (15-66.5) days in the intervention group.

\section{Healthcare utilisation}

In addition to PROMs, three studies reported rates of primary care consultation and/or emergency department attendance within 4-6 weeks following hospital discharge [14, 15, 17]. 
a)

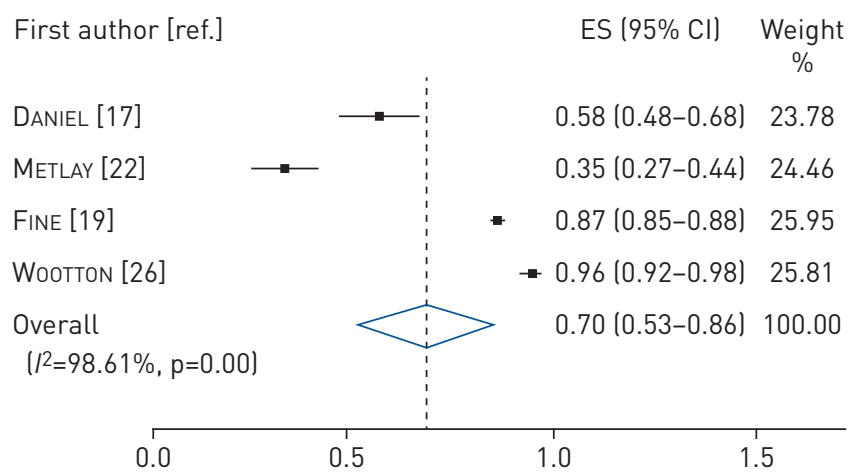

c)

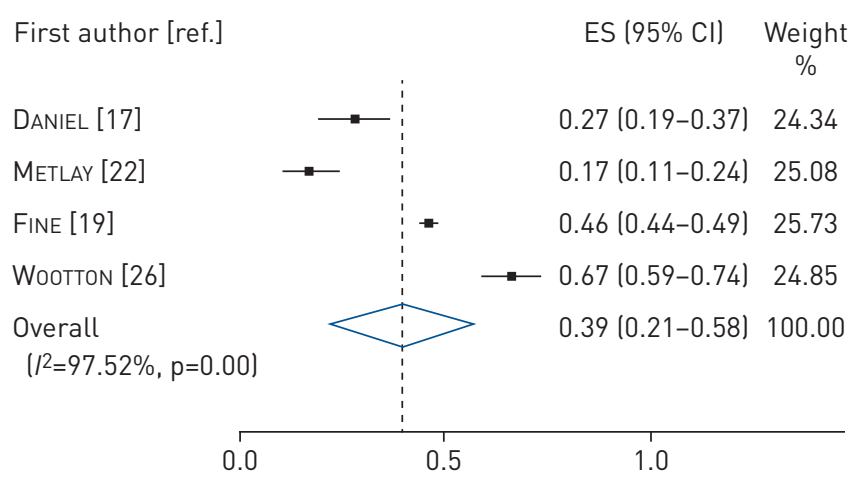

b)

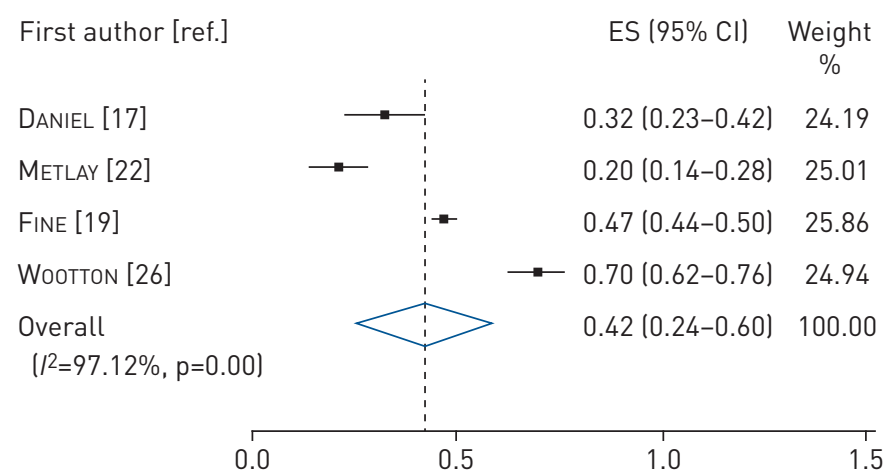

d)

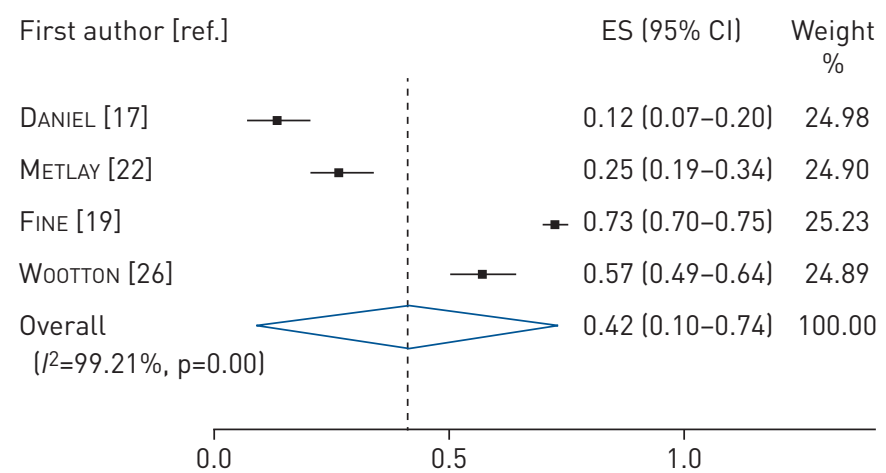

FIGURE 3 Random effects meta-analysis of prevalence of symptoms at 4 weeks following discharge from hospital with community-acquired pneumonia (CAP): a) one or more symptom, b) cough, c) dyspnoea and d) fatigue. ES: effect size. MEtLAY et al. [22] and DaNIEL et al. [17] are studies of patient cohorts restricted to either low/moderate severity CAP (Pneumonia Severity Index classes I-III or CURB-65 (confusion, urea $>7 \mathrm{mmol} \cdot \mathrm{L}^{-1}$, respiratory rate $\geqslant 30$ breaths $\mathrm{min}^{-1}$, blood pressure $<90 \mathrm{mmHg}$ (systolic) or $\leqslant 60 \mathrm{mmHg}$ (diastolic), age $\geqslant 65$ years) score $0-2$ ) or age $<65$ years, by design.

DANIEL et al. [17] found that 59.2\% had consulted primary care and $12.0 \%$ had attended emergency departments within 4 weeks. This compares with $18.0 \%$ and $20.3 \%$ attending primary care and $18.4 \%$ and $21.4 \%$ attending emergency departments in the two studies from ADAmuz et al. [14, 15]. Of those reconsulting a general practitioner, ongoing or new respiratory symptoms were reported by $68.8-75.2 \%$ of patients. The reported reason for emergency department attendance was pneumonia-related symptoms or signs in $47.5-84.6 \%$ of patients $[15,17]$.

\section{Other outcomes}

In relation to quality of life measures, a single Spanish study commented that "SF-36 score at 30 days following hospital discharge remained abnormal when compared to the reference scores for the Spanish population", but no data were reported [16]. Using the EuroQoL EQ-5D health-related quality of life questionnaire, NiCKLER et al. [23] noted that $29 \%$ of participants exhibited a decline in ADLs at 30 days, and that the decline was associated with levels of pro-adrenomedullin and pro-atrial natriuretic peptide.

Physician-assessed clinical cure was noted by BRUNs et al. [6] in $88.9 \%$ of participants 28 days after discharge, although only $41.7 \%$ of patients reported normalisation of symptoms and function (based on the CAP score) at the same time-point. No data were provided on any correlation between clinical cure and CAP score.

\section{Discussion}

This systematic review confirms that a high proportion of patients report substantial symptomatic and functional impairments during the first 6 weeks of recovery following hospitalisation with CAP. These are accompanied by adverse impacts on the performance of "normal activities" (e.g. return to work) and healthcare utilisation. Fatigue, cough and dyspnoea are the commonest symptoms reported. The prevalence of symptoms over the first 6 weeks of recovery varies across studies in accordance with the severity of CAP and age of study cohorts. 
The majority of studies assessed the prevalence of symptoms and functional impairment at specific times (most commonly 4 weeks post-discharge) without reference to baseline prevalence. Given the high proportion of patients with chronic cardiac and respiratory conditions within study cohorts, this approach likely overestimates the contribution of acute pneumonia to the prevalence of patients' symptoms during recovery. That said, studies measuring PROMs at multiple time-points during recovery all reported a declining trend in the prevalence of symptoms over time, up to 42 days post-discharge $[6,18,21,26]$. These findings suggest that many patients continue to have symptoms related to the acute pneumonia episode even at 6 weeks post-discharge.

Fatigue is the commonest symptom to be reported during recovery. The importance of fatigue as a contribution towards reduced functional recovery in CAP has not been widely examined. In a qualitative study of patients with low-severity CAP, both extreme tiredness and need for sleep were reported as prominent symptoms during recovery [28]. Quantitatively, the CAP-symptom score captures the degree of fatigue on a scale of 0-5 [29]. However, these assessments do not adequately reflect the complexity of fatigue as a symptom nor its impact on functional recovery. In other conditions where fatigue is a prominent symptom, validated disease-specific, multidimensional tools have been developed to better quantify and assess fatigue $[30,31]$.

Return to functional activity, measured as return to "normal activities" or ADLs, was consistently reported as taking a median of 15-25 days in included studies. This measure is set alongside other included studies that consistently reported a large proportion of patients failing to resume normal activities within 4 weeks post-discharge. One study, not included in this review, found that at 6 weeks post-discharge, $12 \%$ of elderly persons had required a change of residence, indicating a greater level of dependency [32]. Overall, these studies suggest that the full burden of adverse health outcomes following an episode of CAP is likely to be higher than reflected in measures of symptom resolution alone [33]. Experience from patients recovering from exacerbations of chronic obstructive pulmonary disease, where impaired lung function, exercise capacity and quality of life persists despite apparent symptomatic resolution, provides further support for this view [34-36].

We identified only two relatively small studies that attempted to describe the correlation between PROMs and healthcare reconsultation [14, 17]. These studies suggest that persistence of pneumonia-related symptoms is a major factor in general practitioner reconsultations, while new or worsening comorbid illnesses increase in prominence in relation to emergency department reattendances. An episode of pneumonia may be a marker for frailty or increased susceptibility to illness from non-pneumonia-related factors [37]. A better understanding of the association between PROMs, long-term complications and healthcare reconsultations is necessary if appropriate interventions are to be developed.

\section{Strengths and limitations}

To the best of our knowledge, this is the first systematic review of studies assessing PROMs in recovery from CAP. This review was conducted in accordance with PRISMA guidelines. Eligibility criteria were designed to focus the review on studies of patients with CAP and not on cohorts with hospital-acquired pneumonia or aspiration pneumonia.

We found only a small number of high-quality research studies in this field compared with the large burden of disease. Existing studies are disparate in their study populations (age, comorbid illness and disease severity) and outcome measures (table 2). Importantly, there is a lack of consistency across studies in the choice and application of measurement tools to assess PROMs. The CAP-symptom score is the only available PROM psychometrically validated using a recognised approach, albeit only in an outpatient setting. These variations in outcome measures precluded many studies from the planned meta-analysis; the resulting meta-analysis retained high study heterogeneity. Most notably, the meta-analysis illustrates how, at a given time-point, the severity of CAP and/or age of the study cohort greatly affects estimates of symptom prevalence.

\section{Implications}

There is an important need for researchers to develop and agree on appropriate tools and methodology in the assessment of PROMs during recovery from CAP. BARLOW et al. [9] previously defined core outcomes sets for CAP research, including recommending the CAP-symptom score for use in studies of recovery. Ideally, the CAP-symptom score should be externally validated for hospitalised patients and a multidimensional CAP-specific tool for assessing fatigue developed.

In the meantime, considering the available evidence, we suggest the CAP-symptom score remains the most appropriate tool for the measurement of symptoms. As the prevalence of symptoms remains high for 2-4 weeks post-discharge, and healthcare reconsultation is concentrated to within the same time period, 
we suggest that PROMs should be assessed, at a minimum, twice post-discharge, at around 2 weeks and again at 4 or 6 weeks. To enable analysis of the degree of recovery at these time-points, an assessment at the time of hospital discharge is necessary, together with an assessment of "pre-pneumonia baseline", accepting that the latter is inevitably subject to recall bias. In addition to symptom reporting, we recommend measuring functional recovery, both in terms of quality of life (EQ-5D) and return to normal activities. Ideally, the time needed to return to "baseline" health status within different domains should be measured. These interim suggestions should be refined as new, stronger evidence emerges and our understanding of recovery from CAP increases.

\section{Conclusions}

Morbidity from CAP continues for a sizeable proportion of patients up to at least 6 weeks post-discharge from hospital. There is a large relative lack of high-quality research in this field.

Acknowledgements: The review authors would like to acknowledge the contribution of Dan Wootton (Institute of Infection and Global Health, University of Liverpool and Aintree University Hospitals NHS Foundation Trust, Liverpool, UK) and colleagues for providing additional data to the review beyond that available in their original published manuscript [26].

Conflict of interest: H.J. Pick has nothing to disclose. C.E. Bolton reports grants from GSK and Pfizer, honorarium from Chiesi, and consultancy for Boehringer, outside the submitted work. W.S. Lim has nothing to disclose. T.M. McKeever has nothing to disclose.

\section{References}

1 Trotter CL, Stuart JM, George R, et al. Increasing hospital admissions for pneumonia, England. Emerg Infect Dis 2008; 14: 727-733.

2 National Institute for Health and Care Excellence. Pneumonia in adults; diagnosis and management. 2014. www. nice.org.uk/guidance/cg191 Date last accessed: January 11, 2019.

3 Lim WS, Baudouin SV, George RC, et al. BTS guidelines for the management of community acquired pneumonia in adults: update 2009. Thorax 2009; 64: Suppl. 3, iiil-iii55.

4 Daniel $\mathrm{P}$, Woodhead $\mathrm{M}$, Welham S, et al. Mortality reduction in adult community-acquired pneumonia in the UK (2009-2014): results from the British Thoracic Society audit programme. Thorax 2016; 71: 1061-1063.

5 Welte T, Torres A, Nathwani D. Clinical and economic burden of community-acquired pneumonia among adults in Europe. Thorax 2012; 67: 71-79.

6 Bruns AHW, Oosterheert JJ, El Moussaoui R, et al. Pneumonia recovery; discrepancies in perspectives of the radiologist, physician and patient. J Gen Intern Med 2010; 25: 203-206.

7 Mittl RL Jr, Schwab RJ, Duchin JS, et al. Radiographic resolution of community-acquired pneumonia. Am J Respir Crit Care Med 1994; 149: 630-635.

8 Snijders D, Daniels JM, de Graaff CS, et al. Efficacy of corticosteroids in community-acquired pneumonia: a randomized double-blinded clinical trial. Am J Respir Crit Care Med 2010; 181: 975-982.

9 Barlow GD, Lamping DL, Davey PG, et al. Evaluation of outcomes in community-acquired pneumonia: a guide for patients, physicians, and policy-makers. Lancet Infect Dis 2003; 3: 476-488.

10 Food and Drug Administration. Guidance for industry on community-acquired bacterial pneumonia: developing drugs for treatment. 2014. www.fda.gov/downloads/drugs/guidances/ucm123686.pdf Date last accessed: January $11,2019$.

11 Moher D, Liberati A, Tetzlaff J, et al. Preferred reporting items for systematic reviews and meta-analyses: the PRISMA statement. BMJ 2009; 339: b2535.

12 Wells GA, Shea B, O'Connell D, et al. The Newcastle-Ottawa scale (NOS) for assessing the quality of nonrandomised studies in meta-analyses. 2009. www.ohrica/programs/clinical_epidemiology/oxford.asp Date last accessed: October 5, 2018.

13 Downs SH, Black N. The feasibility of creating a checklist for the assessment of the methodological quality both of randomised and non-randomised studies of health care interventions. J Epidemiol Community Health 1998; 52: $377-384$.

14 Adamuz J, Viasus D, Camprecios-Rodriguez $\mathrm{P}$, et al. A prospective cohort study of healthcare visits and rehospitalizations after discharge of patients with community-acquired pneumonia. Respirology 2011; 16: $1119-1126$.

15 Adamuz J, Viasus D, Simonetti A, et al. Impact of an educational program to reduce healthcare resources in community-acquired pneumonia: the EDUCAP randomized controlled trial. PLoS One 2015; 10: e0140202.

16 Carratala J, Fernandez-Sabe N, Ortega L, et al. Outpatient care compared with hospitalization for community-acquired pneumonia: a randomized trial in low-risk patients. Ann Intern Med 2005; 142: 165-172.

17 Daniel P, Bewick T, McKeever TM, et al. Healthcare reconsultation in working-age adults following hospitalisation for community-acquired pneumonia. Clin Med 2018; 18: 41-46.

18 El Moussaoui R, Opmeer BC, de Borgie CA, et al. Long-term symptom recovery and health-related quality of life in patients with mild-to-moderate-severe community-acquired pneumonia. Chest 2006; 130: 1165-1172.

19 Fine MJ, Stone RA, Singer DE, et al. Processes and outcomes of care for patients with community-acquired pneumonia: results from the Pneumonia Patient Outcomes Research Team (PORT) cohort study. Arch Intern Med 1999; 159: 970-980.

20 Labarere J, Stone RA, Obrosky DS, et al. Comparison of outcomes for low-risk outpatients and inpatients with pneumonia: a propensity-adjusted analysis. Chest 2007; 131: 480-488.

21 Marrie TJ, Lau CY, Wheeler SL, et al. Predictors of symptom resolution in patients with community-acquired pneumonia. Clin Infect Dis 2000; 31: 1362-1367. 
22 Metlay JP, Atlas SJ, Borowsky LH, et al. Time course of symptom resolution in patients with community-acquired pneumonia. Respir Med 1998; 92: 1137-1142.

23 Nickler M, Schaffner D, Christ-Crain M, et al. Prospective evaluation of biomarkers for prediction of quality of life in community-acquired pneumonia. Clin Chem Lab Med 2016; 54: 1831-1846.

24 Sharma G, Han L, Quagliarello V. Short- and long-term functional outcome of hospitalized older adults with community-acquired pneumonia. Infect Dis Clin Pract 2006; 14: 365-368.

25 Uranga A, Espana PP, Bilbao A, et al. Duration of antibiotic treatment in community-acquired pneumonia: a multicenter randomized clinical trial. JAMA Intern Med 2016; 176: 1257-1265.

26 Wootton DG, Dickinson L, Pertinez H, et al. A longitudinal modelling study estimates acute symptoms of community acquired pneumonia recover to baseline by 10 days. Eur Respir J 2017; 49: 1602170.

27 Wyrwich KW, Yu H, Sato R, et al. Observational longitudinal study of symptom burden and time for recovery from community-acquired pneumonia reported by older adults surveyed nationwide using the CAP Burden of Illness Questionnaire. Patient Relat Outcome Meas 2015; 6: 215-223.

28 Baldie DJ, Entwistle VA, Davey PG. The information and support needs of patients discharged after a short hospital stay for treatment of low-risk community acquired pneumonia: implications for treatment without admission. BMC Pulm Med 2008; 8: 11

29 Lamping DL, Schroter S, Marquis P, et al. The community-acquired pneumonia symptom questionnaire: a new, patient-based outcome measure to evaluate symptoms in patients with community-acquired pneumonia. Chest 2002; 122: 920-929.

30 Butt Z, Lai JS, Rao D, et al. Measurement of fatigue in cancer, stroke, and HIV using the Functional Assessment of Chronic Illness Therapy - Fatigue (FACIT-F) scale. J Psychosom Res 2013; 74: 64-68.

31 Comi G, Leocani L. Assessment, pathophysiology and treatment of fatigue in multiple sclerosis. Expert Rev Neurother 2002; 2: 867-876.

32 Gladman J, Barer D, Venkatesan P, et al. The outcome of pneumonia in the elderly: a hospital survey. Clin Rehabil 1991; 5: 201-205.

33 Waterer G. Recovery from community acquired pneumonia: the view from the top of the iceberg. Eur Respir J 2017; 49: 1700571

34 Seemungal TA, Donaldson GC, Bhowmik A, et al. Time course and recovery of exacerbations in patients with chronic obstructive pulmonary disease. Am J Respir Crit Care Med 2000; 161: 1608-1613.

35 Miravitlles M, Ferrer M, Pont A, et al. Effect of exacerbations on quality of life in patients with chronic obstructive pulmonary disease: a 2 year follow up study. Thorax 2004; 59: 387-395.

36 Cote CG, Dordelly LJ, Celli BR. Impact of COPD exacerbations on patient-centered outcomes. Chest 2007; 131: 696-704.

37 Dang TT, Eurich DT, Weir DL, et al. Rates and risk factors for recurrent pneumonia in patients hospitalized with community-acquired pneumonia: population-based prospective cohort study with 5 years of follow-up. Clin Infect Dis 2014; 59: 74-80. 